\title{
Frequency of simultaneous carriage of Neisseria gonorrhoeae and Neisseria meningitidis
}

\author{
K. ØDEGAARD AND T. W. GEDDE-DAHL
}

From the Departments of Bacteriology and Epidemiology, National Institute of Public Health, Oslo, Norway

SUMMARY Gonococci were found by culture in 112 of 442 patients suspected of having gonorrhoea. Meningococci were cultured from the pharynx more than twice as often in patients harbouring gonococci $(26 \%)$ than in patients from whom gonococci could not be cultured $(11 \%)$. These results could indicate an individual susceptibility to Neisseria organisms or they could be caused by a difference in the behaviour of patients with and without gonorrhoea, the conduct of the former making them not only more likely to be infected with Neisseria gonorrhoeae but possibly more exposed to Neisseria meningitidis as well.

\section{Introduction}

Willcox et al. (1977) found that of 150 pharyngeal cultures from patients who were examined because they had a history of oral sexual intercourse Neisseria meningitidis was isolated nearly six times more often in patients with genital gonorrhoea than in those whose genital cultures gave negative results. On the other hand, Neisseria gonorrhoeae was found $21 / 2$ times more often in those who carried the meningococcus in the pharynx than in those who did not. Willcox et al. suggest that if these findings could be confirmed they could indicate an individual susceptibility to the acquisition of Neisseria organisms that would merit further investigation.

Rufli (1978), in a series of 2412 patients, isolated $N$. meningitidis $2 \frac{1}{2}$ times more often from patients with genital gonorrhoea than from patients without and genital $N$. gonorrhoeae almost twice as often from patients with tonsillar $N$. meningitidis than from patients without. The aim of the present paper is to see if these findings could be confirmed.

\section{Patients and methods}

Unselected patients suspected of having gonorrhoea who visited the Department of Venereal Diseases at the Oslo Board of Health from the middle of September to the end of October 1978 were included

Address for reprints: Dr K. Ødegaard, National Institute of Public Health, Geitmyrsveien 75, Oslo 1, Norway

Received for publication 7 February 1979 in the study. On their first visit, samples were taken from the urethra, cervix, rectum, and pharynx in women and from the urethra and pharynx in men. In a few cases rectal samples were also taken from men.

The samples were taken with charcoalimpregnated swabs, which were placed in solid Stuart's transport medium, and sent to the laboratory, where they were cultured from two to five hours later. The specimens were streaked on selective chocolate agar medium containing colistin, lincomycin, trimethoprim, and nystatin (סdegaard, 1971; Odegaard et al., 1975) and incubated at $37^{\circ} \mathrm{C}$ in an atmosphere containing $5 \% \mathrm{CO}_{2}$. The gonococci and the meningococci were identified on the basis of colonial and microscopical examination, oxidase and fermentation reactions, as well as growth requirements. Glucose, maltose, lactose, and sucrose were used for fermentation tests. Nutrient agar incubated at $22^{\circ} \mathrm{C}$ and Dubos oleic acid agar medium incubated at $36^{\circ} \mathrm{C}$ in an atmosphere containing $5 \%$ $\mathrm{CO}_{2}$ were used for growth requirements.

The meningococci were distinguished from the gonococci by their ability to ferment maltose and to grow on Dubos oleic acid agar medium. Both organisms failed to ferment sucrose or lactose or to grow on nutrient agar at $22^{\circ} \mathrm{C}$.

\section{Results}

Samples from 442 patients (149 women and 293 men) were collected. $N$. gonorrhoeae was isolated by culture in $112(25 \%)$ patients (42 women and 70 men), while $N$. meningitidis was isolated in $64(14 \%)$ 
patients (22 women and 42 men). In four patients $N$. gonorrhoeae was cultured from the pharynx. In none of the cases was $N$. meningitidis cultured from the genital or rectal samples.

In 330 patients with negative culture results for $N$. gonorrhoeae, $N$. meningitidis was cultured from 35 (11\%) (12 women and $23 \mathrm{men})$, whereas in the 112 patients with positive culture results for $N$. gonorrhoeae, $N$. meningitidis was cultured from 29 (26\%) (10 women and 19 men). In 378 patients with negative culture results for $N$. meningitidis, gonococci were found in $83(22 \%)$ ( 32 women and 51 men) whereas in the 64 patients from whom $N$. meningitidis was cultured, gonococci were found in $29(45 \%)$ (10 women and 19 men). The results are shown in the Table.

Thus, a highly significant correlation appears to exist between the occurrence of gonococci and meningococci.

The relative risk of harbouring meningococci when gonococci are found is 2.44 with an approximate $95 \%$ confidence interval of $1 \cdot 4-4 \cdot 2$.

Table Culture results for Neisseria gonorrhoeae and Neisseria meningitidis in 442 patients

\begin{tabular}{llll}
\hline & \multicolumn{2}{l}{ Culture results } \\
\cline { 2 - 3 } & \multicolumn{2}{l}{ N. meningitidis } & \\
\cline { 2 - 3 } Culture results & Positive & Negative & Total \\
\hline N. gonorrhoeae & & & \\
Positive & 29 & 83 & 112 \\
Negative & 35 & 295 & 330 \\
Total & 64 & 378 & 442 \\
\hline
\end{tabular}

$x_{1}^{2}=14.6 \mathrm{P}<0.001$

\section{Discussion}

The highly significant correlation between the occurrence of gonococci and meningococci is in agreement with the findings of Willcox et al. (1977) and Rufli (1978).

The results could indicate either an individual susceptibility to the acquisition of Neisseria organisms or a difference in behaviour of patients with and without gonorrhoea, the conduct of the former making them not only more likely to be infected with $N$. gonorrhoeae but possibly more exposed to the acquisition of $N$. meningitidis as well. Both possibilities might also co-exist.

The findings of Lee et al. (1978) of recurrent bacteraemic infections due to Neisseria associated with familial deficiency of the seventh component of complement indicate, however, that individual susceptibility factors may play a part.

\section{References}

Lee, T. J., Utsinger, P. D., Snyderman, R., Yount, W. J., and Sparling, P. S. (1978). "Familial deficiency of the seventh component of complement associated with recurrent bacteremic infections due to Neisseria. Journal of Infectious Diseases, 138, 359-367.

Odegaard, K. (1971). Trimethoprim for the prevention of overgrowth by swarming Proteus in the cultivation of gonococci. Acta Pathologica et Microbiologica Scandinavica, Section B, 79, 545-548.

Odegaard, K., Solberg, O., Lind, J., Myhre, G., and Lyland, B. (1975). Lincomycin in selective medium for the isolation of Neisseria gonorrhoeae. Acta Pathologica et Microbiologica Scandinavica, Section B, 83, 301-304.

Rufli, Th. (1978). Which Neisseria? British Journal of Venereal Diseases, 54, 352.

Willcox, R. R., Spencer, R. C., and Ison, C. (1977). Which Neisseria? British Journal of Venereal Diseases, 53, 384-397. 